\title{
La influencia del sexo y el rol en las interrupciones producidas en tertulias periodísticas sobre política
}

\author{
Marina González-Sanz - Universidad de Sevilla \\ mgsanz@us.es
}

Rebut / Received: 20-7-17

Acceptat / Accepted: 18-9-17

\begin{abstract}
Resum. La influència del sexe i el rol en les interrupcions produïdes en tertúlies periodístiques sobre política. Dins de l'àmbit d'estudi que tracta les diferències lingüístiques existents entre la parla dels homes i les dones, són moltes les investigacions realitzades al voltant de la manifestació de cortesia verbal atenent el factor sexe del parlant. En aquesta ocasió, ens centrem en el cas específic de la interrupció, fenomen en el qual els estudis inicials semblaven confirmar l'estereotip, vigent en les cultures occidentals, que consistia a afirmar que les dones parlaven en excés i que els homes les interrompien. En aquest sentit, en aquest treball pretenem ensenyar la correlació que s'estableix entre la realització en la conversa d'interrupcions de diferent naturalesa i els factors sexe i rol del parlant en un tipus discursiu específic: la tertúlia periodística de tema polític, on es produeix un sistema, en general, lliure pel que fa a la distribució del torn de parla.
\end{abstract}

Paraules clau: interrupció, tertúlia política, sistema d'alternança de torns, anàlisi del discurs, discurs mediàtic.

\begin{abstract}
Influence of gender and role on interruptions in political talk shows. Several works have been carried out about linguistic differences between men and women talk and, specifically, about how they use polite and impolite strategies. In this article, we aim to focus on interruption, an impolite strategy whose appearance in conversation was initially explained by some authors as the consequence of the fact that, in Western communities, women spoke so much that men interrrupted them. In this sense, we intend to show the correlation between different kinds of interruptions and gender and role variables in a specific discourse type, the political talk show, in which turn-talking generally takes place freely.
\end{abstract}


Key words: interruption, political talk show, turn-taking system, discourse analysis, media discourse.

\section{Introducción}

La interrupción constituye uno de los fenómenos más analizados en el marco del análisis del discurso oral y los estudios de la cortesía ${ }^{1}$. Si bien es cierto que en las primeras aproximaciones que se llevaron a cabo únicamente se contemplaban los intercambios ideales, concibiendo la interrupción fundamentalmente como una violación de la correcta alternancia de turnos de habla (Duncan, 1972; Sacks, Schegloff y Jefferson, 1974), trabajos posteriores han ido colocando al fenómeno en un punto central, hasta demostrar que su aparición forma parte de la norma de muchas conversaciones cotidianas (Bañón, 1997; Briz, 1998; Cordisco, 2003; Gallardo, 1993; Hidalgo, 1998; Ridao, 2009).

También ha sido ampliamente debatida la función que este fenómeno desempeña en el discurso: durante décadas se explotó la vinculación existente entre interrupción y poder (Fraser y Nolen, 1981), de manera que interrumpir constituía un recurso más en la manifestación de descortesía (Haverkate, 1994). Contrarias a esta idea surgieron algunas voces discordantes que reivindicaban la naturaleza polifuncional del fenómeno (véase, entre otros, Goldberg, 1990), al incluir en el análisis no solo interrupciones intrusivas y descorteses, sino también otras de naturaleza cooperativa (Ávila, 1997; Bengoechea, 1993; Briz, 1998; Cordisco, 2003; Gallardo, 1993; Hidalgo, 1998; Hutchby, 1992; Murata, 1994; Tannen, 1994). Además de la polifuncionalidad intrínseca que presenta la interrupción, el análisis de este fenómeno debe realizarse desde una perspectiva discursiva y atender necesariamente a la interacción en la que se produce, dado que se encuentra altamente condicionado por factores tales como la situación de comunicación, el género discursivo (Bolívar, 2010), la norma en cuanto a la gestión interactiva (López Serena y Méndez, 2009; Watts, 1991), la interpretación que hacen los propios interlocutores del fenómeno (Ávila, 1997), las propiedades específicas de cada cultura (Fant, 1989, 1996; Haverkate, 1994; Johnson, 2006; Murata, 1994; Poyatos, 1980) o el rol y el sexo de los hablantes implicados en el proceso, variables en las que nos centramos en este trabajo. En lo relativo al último parámetro, los estudios iniciales parecieron confirmar un estereotipo, vigente en las culturas occidentales, que consiste en afirmar que las mujeres hablan en exceso, y que los hombres las interrumpen ${ }^{2}$. El trabajo fundador de esta tesis fue realizado por Zimmerman y West (1975), que comenzaron una línea de

1. Véase Hutchby (1992), Murata (1994) y Roger, Bull y Smith (1988) para obtener una panorámica internacional del fenómeno. En España el estudio de la interrupción comienza años más tarde (Bañón, 1996 y 1997; Bengoechea, 1993; Carbó, 1992; Cestero, 1994a y 1994b; Gallardo, 1993; Hidalgo, 1998).

2. Véase García Mouton (1999), James y Clarke (1994) o Tannen (1994) para esta idea. 
estudio continuada por otros muchos autores en la década de los 70 y 80 . Todos ellos concluyen que, efectivamente, los hombres interrumpen más a sus interlocutoras que a sus interlocutores (Holmes, 1995; West y Zimmerman, 1987) 3 $^{3}$ Sin embargo, estos análisis parecen no atender a las diferencias que se producen en contextos comunicativos específicos (Lozano, 1995), donde los datos apuntan a conclusiones contradictorias (Beattie, 1981; Cestero, 2007).

Así las cosas, en este trabajo pretendemos mostrar la correlación que se establece entre la realización de interrupciones, fundamentalmente descorteses pero también corteses y neutras, y los factores rol y sexo del hablante, de manera que podamos comprobar si, como ocurre en otros contextos de comunicación, en la tertulia periodística de tema político también se producen más interrupciones procedentes de los interlocutores masculinos o si, por el contrario, las contertulias interrumpen con más frecuencia que los participantes masculinos. Asimismo, incluiremos en el análisis las variables rol y sexo del interlocutor, para observar si, como parece ocurrir en otras situaciones de comunicación, las mujeres son más interrumpidas por sus interlocutores (ya sean masculinos o femeninos) que los hombres.

\section{Corpus y metodología}

Para alcanzar nuestros objetivos de investigación, hemos partido de un estudio global sobre la tertulia periodística de tema político, que ahonda en su caracterización y, específicamente, en el funcionamiento que presenta la descortesía en este tipo discursivo (González-Sanz, 2017). Para esta investigación específica, hemos seleccionado un corpus formado por un total de 8 transcripciones de tertulias radiofónicas (4 programas) y televisivas (4 programas), que fueron emitidas entre los años 2009 y $2013^{4}$. La equilibrada presencia de material de radio y de televisión garantiza la fiabilidad de las conclusiones 5 .

El marco teórico del que partimos en este estudio es la lingüística pragmática, tal y como aparece descrita en Fuentes (2000). De manera específica, nos hemos valido de las herramientas desarrolladas en el seno de los estudios sobre cortesía y descortesía verbal, en especial sobre el funcionamiento de la interrupción, para lo que hemos partido del modelo teórico propuesto por Bañón (1997), así como de los análisis efectuados desde la perspectiva de la pragmática sociocultural (Bravo y Briz, 2004; Fant, 1989).

3. Una de las explicaciones aducidas radica en la concepción negativa que tienen las mujeres de la interrupción, relacionada con una clara violación de las normas interaccionales, diferente a la tolerancia hacia el fenómeno que parecen mostrar los hablantes masculinos (Bresnahan y Cai, 1996).

4. En concreto, se trata de los programas La noria (19/09/2009), El gato al agua (15/09/2011), El programa de Ana Rosa (20/12/2010), Espejo público (30/11/2009), Herrera en la Onda (30/05/2011), Hoy por hoy (01/03/2013), La linterna (23/05/2011) y La noche de César (03/07/2012).

5. En términos estadísticos, el material procedente de tertulias televisivas se corresponde con un 57\%, mientras que el correspondiente a programas radiofónicos alcanza el $43 \%$. 
Para realizar el estudio cuantitativo, se ha llevado a cabo un análisis descriptivo de las principales variables cualitativas del estudio, para lo que se han calculado las frecuencias absolutas y relativas. Para ver la relación entre las variables se han utilizado los test de comparación de proporciones para muestras independientes, en concreto la prueba de chi-cuadrado. El nivel de riesgo alfa aceptado para todos los contrastes de hipótesis se ha establecido en 0.05 . El programa estadístico utilizado para realizar dichos análisis ha sido Epidat 3.1 (DXSP-OPS, 2006).

\section{La interrupción en la tertulia periodística de tema político}

La tertulia periodística de tema político constituye una modalidad de talk-show, de gran éxito en nuestro país, en la que varios contertulios tratan asuntos relativos a la actualidad política nacional y, en menor medida, internacional. La interacción se encuentra regulada por un moderador que introduce y gestiona el tema y distribuye los turnos de habla, en especial en las fases iniciales y finales de la tertulia. En la etapa central del programa se produce una interacción esencialmente libre, en la que no se establece una distribución previa del turno de habla y las sucesivas intervenciones se guían por el método de autoselección.

Se trata de una situación comunicativa de gran complejidad, que combina rasgos propios del discurso político (dimensión argumentativa, tratamiento ideológico del tema, eufemismos) con características vinculadas al discurso mediático (estructura de la interacción, registro coloquial o caracterización enunciativa). El fin que persiguen los participantes de la interacción posee, en consecuencia, una doble naturaleza mediáticoideológica: pretende, por una parte, aumentar o, como mínimo, mantener los niveles de audiencia; por otra parte, persigue convencer a los espectadores o a los oyentes de seguir una determinada opción ideológica. Además, este tipo discursivo presenta un rasgo caracterizador, común a muchos textos políticos y mediáticos: la descortesía verbal (González-Sanz, 2014). En concreto, en las interacciones que forman parte de las tertulias analizadas es común encontrar descalificaciones, manifestaciones de desacuerdo e interrupciones, fenómeno del que nos ocupamos en este trabajo.

De entre los múltiples enfoques que existen para abordar el análisis de la interrupción, nos hemos decantado por considerarlo como un proceso semiocomunicativo, de naturaleza interactiva, natural a intercambios comunicativos de diverso tipo (Bañón, 1997). En el caso específico de las tertulias que analizamos, la sucesión de hablantes en turno de habla, que se lleva a cabo fundamentalmente mediante el mecanismo de autoselección, se presenta conflictiva en el 32\% de los casos.

(1) Moderador: Mari Antonia $\downarrow$ por favor Mari Antonia $\downarrow$

Enric Sopena: a la señora Merkel le faltan unas elecciones no [sé si te has entera $(\mathrm{d}) \mathrm{o}]$

Ma Antonia Iglesias: [bueno a ver] 


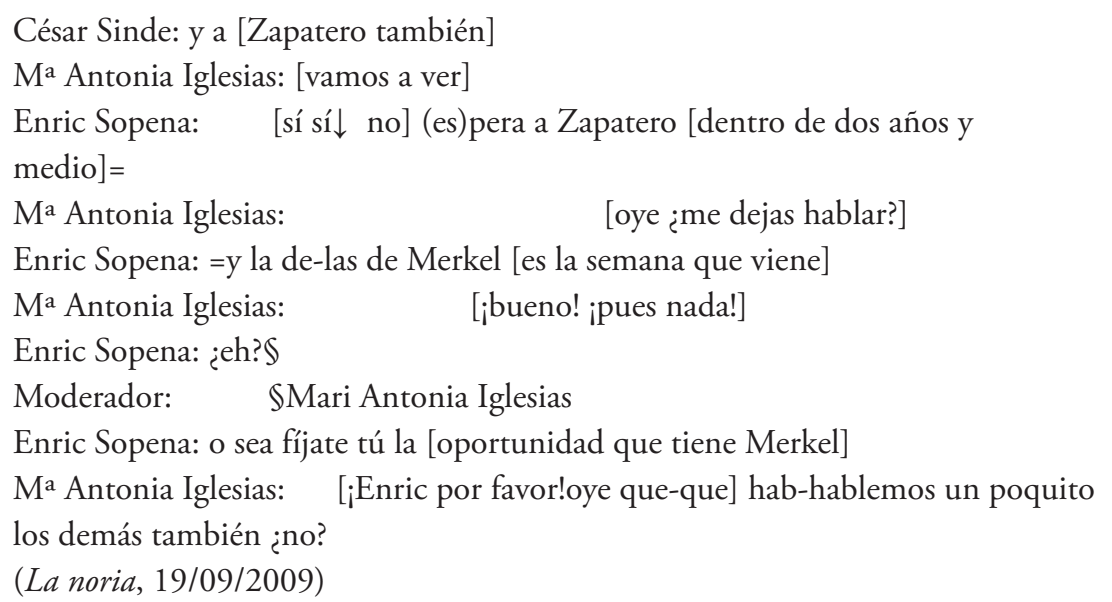

Como podemos observar, en el ejemplo (1) se produce una verdadera lucha por obtener el turno de habla, a través de diferentes mecanismos, tales como el solapamiento, la repetición, el uso de marcadores de inicio de intervención, etc. Estos procesos interruptores pueden aparecer en cualquier punto del desarrollo de la tertulia e involucrar no solo a tertulianos sino también al moderador del programa. No nos extraña, por otra parte, que la interrupción tenga una presencia tan elevada en el corpus que analizamos: por un lado, se trata de interacciones polilogales, más complejas que los intercambios protagonizados únicamente por dos hablantes (Beattie, 1981); por otro lado, desde un punto de vista cultural, los hispanohablantes peninsulares presentan un alto grado de tolerancia a las interrupciones (Fant, 1989), por lo que no se precisa un elevado nivel de consenso para continuar una conversación.

El corpus sobre el que hemos realizado esta investigación está compuesto por 2.859 intervenciones, con la siguiente distribución de hablantes según rol y sexo: 5 moderadores hombres (en adelante $\mathrm{MH}$ ), 3 moderadoras mujeres (MM), 20 tertulianos hombres (TH) y 11 tertulianas mujeres (TM). El nivel de participación de cada instancia discursiva (en cantidades absolutas y porcentuales) se ofrece en la Tabla 1:

TABLA I. INTERVENCIONES EMITIDAS POR CADA HABLANTE, SEGÚN LOS PARÁMETROS ROL Y SEXO

\begin{tabular}{|l|c|c|}
\hline & $\begin{array}{c}\text { Número de } \\
\text { intervenciones }\end{array}$ & Porcentaje \\
\hline $\mathrm{MH}$ & 529 & $18,5 \%$ \\
\hline $\mathrm{MM}$ & 112 & $3,92 \%$ \\
\hline $\mathrm{TH}$ & 1380 & $48,27 \%$ \\
\hline $\mathrm{TM}$ & 838 & $29,31 \%$ \\
\hline
\end{tabular}


El número de interrupciones producidas por cada tipo de hablante se muestra en las siguientes tablas, en las que hemos diferenciado entre los programas de televisión (TV) (Tabla 2) y radio $(\mathrm{RD})$ (Tabla 3):

TABla 2. Distribución DE INTERRUPCIONES EMITIDAS POR CADA TIPO DE habLANTE (TV)

\begin{tabular}{|l|l|c|c|c|c|}
\hline \multicolumn{2}{|c|}{} & Frecuencia & Porcentaje & $\begin{array}{c}\text { Porcentaje } \\
\text { válido }\end{array}$ & $\begin{array}{c}\text { Porcentaje } \\
\text { acumulado }\end{array}$ \\
\hline \multirow{4}{*}{ Válidos } & Moderador hombre & 83 & 5,6 & 12,0 & 12,0 \\
\cline { 2 - 6 } & Moderador mujer & 39 & 2,6 & 5,6 & 17,7 \\
\cline { 2 - 6 } & Tertuliano hombre & 398 & 26,8 & 57,6 & 75,3 \\
\cline { 2 - 6 } & Tertuliano mujer & 171 & 11,5 & 24,7 & 100,0 \\
\cline { 2 - 6 } & Total & 691 & 46,6 & 100,0 & \\
\hline Perdidos & Sistema & 792 & 53,4 & & \\
\hline \multicolumn{1}{|l|}{ Total } & 1483 & 100,0 & & \\
\hline
\end{tabular}

TABla 3. Distribución de interrupciones emitidas por Cada tipo de hablante (RD)

\begin{tabular}{|l|l|c|c|c|l|}
\hline \multicolumn{2}{|c|}{} & Frecuencia & Porcentaje & $\begin{array}{c}\text { Porcentaje } \\
\text { válido }\end{array}$ & $\begin{array}{l}\text { Porcentaje } \\
\text { acumulado }\end{array}$ \\
\hline \multirow{3}{*}{ Válidos } & Moderador hombre & 90 & 6,5 & 20,3 & 20,3 \\
\cline { 2 - 6 } & Moderador mujer & 11 &, 8 & 2,5 & 22,7 \\
\cline { 2 - 6 } & Tertuliano hombre & 198 & 14,4 & 44,6 & 67,3 \\
\cline { 2 - 6 } & Tertuliano mujer & 145 & 10,5 & 32,7 & 100,0 \\
\cline { 2 - 6 } & Total & 444 & 32,3 & 100,0 & \\
\hline Perdidos & Sistema & 932 & 67,7 & & \\
\hline \multicolumn{2}{|l|}{ Total } & 1376 & 100,0 & & \\
\hline
\end{tabular}

Desde el punto de vista del medio, se detectan diferencias significativas entre las interrupciones producidas en radio y las correspondientes a la televisión: en prácticamente todas las instancias discursivas, a excepción de las moderadoras mujeres ${ }^{6}$, contamos con mayores índices de producción de interrupciones en televisión que en radio. Así ocurre con los moderadores (39,34\% en televisión frente a $28,30 \%$ en radio), los tertulianos hombres (48,89\% en televisión y 34,98 en radio) y, por último, las tertulianas mujeres ( $45,36 \%$ en televisión frente a $31,45 \%$ correspondiente a la radio). En todos estos casos

6. De la que contamos con una única representante en el medio radiofónico, lo que nos impide generalizar las conclusiones obtenidas acerca de esta instancia discursiva en concreto. 
el P valor ha sido inferior a 0,05 y ello que constata que nos encontramos ante diferencias significativas en términos estadísticos.

Ahora bien, como señalábamos antes, no todas las interrupciones poseen el mismo valor en la interacción. En términos de cortesía verbal, si bien no podemos aceptar el valor cortés de la interrupción, esto no impide que podamos distinguir entre interrupciones no descorteses e interrupciones descorteses. Dado que la interrupción constituye un fenómeno pragmático, altamente dependiente de la incidencia de factores extralingüísticos, hemos optado también por atender a las diferencias jerárquicas existentes entre los interlocutores implicados ${ }^{7}$ para describir el proceso interruptor ante el que nos encontramos en cada caso. Así, en interacciones de tipo $\mathrm{M}>\mathrm{T}$, esto es, aquellas en las que un moderador interrumpe el discurso de un tertuliano, hemos considerado descorteses únicamente aquellas interrupciones destinadas a introducir la expresión de una discrepancia con la opinión manifestada en la intervención interrumpida.

(2) Ester Esteban: tiene voluntad $\downarrow$ mañana mismo hay elecciones $\downarrow$ o sea el que con- el que tiene capacidad [para decir]

Fernando Rayón: $\quad$ [no $\downarrow$ ies él!!

Ester Palomera: ¡claro!

Federico Quevedo: claro

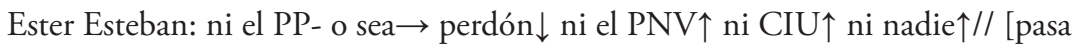
por esto $\downarrow$ entonces]

Moderador: [pues anoche ha declarado] que esa voluntad no la tiene $\downarrow$

[entonces]

Federico Quevedo: [bueno yo no lo tengo tan claro $\downarrow$ eee $\uparrow$ ]

Fernando Rayón: [porque Zapatero no tiene $\downarrow /$ porque Zapatero es el el [el que se queda en evidencia $\downarrow$ siempre]

Ester Esteban: [ayer lo dejó clarísimo]

(La linterna, 23/05/2011)

En (2), el moderador de la tertulia radiofónica de La linterna interrumpe el discurso desarrollado por la contertulia E. Esteban para mostrar una opinión discordante con ella. A pesar de la diferencia jerárquica de rol que existe entre ambos interlocutores, la expresión de una opinión discordante constituye un acto descortés por la amenaza que conlleva sobre la imagen de la tertuliana. Por el contrario, el resto de interrupciones producidas por moderadores sobre el discurso de tertulianos son no descorteses, debido a que la diferencia de rol permite al moderador interrumpir el discurso del resto de participantes para regular la interacción o colaborar en la construcción de su discurso sin que la imagen de estos resulte dañada. Estas interrupciones del moderador sobre el

7. Véase Bañón (1997). 
discurso de tertulianos se dividen, a su vez, en interrupciones de tipo neutro (3), fruto del cumplimiento del rol del moderador, encargado de regular los turnos de habla y dinamizar la interacción) o cooperativo (4), destinadas a colaborar en la construcción del discurso del hablante interrumpido, proporcionando una palabra necesaria que el hablante no localiza, realizando preguntas para que el interlocutor desarrolle la información u opinión que proporciona, etc.

(3) Carlos Rodríguez: yo creo que el movimiento del quince eme tiene-tiene dos posibilidades SEGÚN sea como nos plantearon al principio $\downarrow$ es decir $\$$

Moderador: \$nos quedan treinta segundos doctor $\uparrow$

Carlos Rodríguez: ah ipues!- o bien eres independiente $\uparrow$ o bien eres de izquierda $\downarrow /$ si eres independiente es como el movimiento argentino ese de ¡QUE SE VAYAN TODOS!/ que os acordáis que al final pues quedó en nada $\downarrow /$ y si es de- entonces va a desaparecer $\downarrow$ pero si es de verdad de izquierdas y tiene alguna relación con la izquierda entonces va a perdurar sobre todo ahora en época electoral

(Herrera en la Onda, 30/05/2011)

(4) Ester Palomera: $=[\mathrm{y}$ no] quedarnos solo en LA [CONVOCATORIA $]=$

Federico Quevedo: [sí pero]

Ester Palomera: =DE ELECCIONES ANTICIPADAS $\downarrow /$ porque hoy por hoy eso parece que [no va a ser]

Moderador: [que no sale $\downarrow$ ] no sale $\downarrow$ no sale $\downarrow$ [no sale]

(La linterna, 23/05/2011)

Así, en (3), el moderador interrumpe el discurso del contertulio para recordarle que el programa se encuentra próximo a su fin, mientras que en (4) apoya con su intervención el discurso de E. Palomera.

En lo relativo al esquema interactivo establecido entre tertulianos, encontramos igualmente interrupciones efectuadas con propósito cooperativo (el ejemplo (5) muestra cómo la contertulia A. Estrada completa el enunciado que M. Á. Rodríguez no es capaz de finalizar por no hallar el término preciso) o descortés (6), introduciendo, por ejemplo, discrepancias con respecto a la posición mantenida por el interlocutor interrumpido:

(5) Eloísa de Dios: [pero si hay una ley] de libertad religiosa $\uparrow /$ no- lo que no puedes hacer es contradecir [esa ley dentro del] =

Amparo Estrada:

[o(eso digo yo)o]

Eloísa de Dios: =mismo país $\$$

Miguel Ángel Rodríguez: $\Phi_{i} y a !$ lo que pasa es que ee-e frente a una ley -de libertad religiosa y OTRAS个/ está el principio de de contraprestación- no se dice contraprestación [pero ¡en fin!]
Amparo Estrada:
[de reciproci]dad 
Miguel Ángel Rodríguez: de-de reciprocidad ¿̨no?

Amparo Estrada: pero no puede existir reciprocidad ee- [en-]

Miguel Ángel Rodríguez:

[¿por] qué?

(Espejo público, 30/11/2009)

(6) Antonio Miguel Carmona: digamos que estas pueden ser las clases más

adineradas adineradas $\downarrow$ / que eluden- que pueden pagar el impuesto del IRPF pero tienen capacidad de elusión fiscal $\downarrow$ / y luego hay una tercera copa que no existe

Alejo Vidal-Quadras: qué tienen capacidad $\uparrow$ ¿cómo?

Antonio Miguel Carmona: de elusión fiscal

Alejo Vidal-Quadras: ¡ah! ¡sí?

Antonio Miguel Carmona: sí $\downarrow$ sí $\downarrow$ ¡hombre!/ [te lo voy a decir yo $\downarrow$ ]

Alejo Vidal-Quadras: ¡¿[LA CLASE MEDIA] TIENE

CAPACIDAD DE ELUSIÓN FISCAL?!

Antonio Miguel Carmona: no $\downarrow$ yo no he dicho eso $\downarrow$ [déjeme-déjeme terminar]

Alejo Vidal-Quadras:

[¿elusión? ¿elusión?]

(El gato al agua, 15/096/2011)

En este último caso nos encontramos ante un acto amenazante para la imagen de A. M. Carmona, cuyo discurso ha recibido una opinión disentiva que además ha interrumpido su exposición.

Las siguientes tablas ofrecen, en términos cuantitativos, los tipos de interrupciones localizados en el corpus que manejamos, tanto en televisión (Tabla 4) como en radio (Tabla 5):

Tabla 4. Distribución de interrupciones SEgún SU NATURAleza ATENDIENDO A LAS VARIABLES ROL Y SEXO DEL HABLANTE (TV)

\begin{tabular}{|l|c|c|c|c|}
\hline \multirow{2}{*}{ Hablante } & \multicolumn{3}{|c|}{ Naturaleza de la interrupción } & \multirow{2}{*}{ Total } \\
\cline { 2 - 5 } & Neutras & Cooperativas & Descorteses & \\
\hline \multirow{3}{*}{ Moderador hombre } & 45 & 21 & 17 & 83 \\
\cline { 2 - 5 } & $54,2 \%$ & $25,3 \%$ & $20,5 \%$ & $100,0 \%$ \\
\hline \multirow{2}{*}{ Moderador mujer } & 18 & 6 & 15 & 39 \\
\cline { 2 - 5 } & $46,2 \%$ & $15,4 \%$ & $38,5 \%$ & $100,0 \%$ \\
\hline \multirow{3}{*}{ Tertuliano hombre } & 0 & 13 & 385 & 398 \\
\cline { 2 - 5 } Tertuliano mujer & $0 \%$ & $3,3 \%$ & $96,7 \%$ & $100,0 \%$ \\
\cline { 2 - 5 } & 0 & 3 & 168 & 171 \\
\hline \multirow{2}{*}{ Total } & 63 & $1,8 \%$ & $98,2 \%$ & $100,0 \%$ \\
\cline { 2 - 5 } & $9,1 \%$ & $6,2 \%$ & $84,7 \%$ & $100,0 \%$ \\
\hline
\end{tabular}


TABla 5. Distribución de INTERRUPCiONES SEgún SU NATURALEZa ATENDIENDO A LAS VARIABLES ROL Y SEXO DEL HABLANTE (RD)

\begin{tabular}{|l|c|c|c|c|}
\hline \multirow{2}{*}{ Hablante } & \multicolumn{3}{|c|}{ Naturaleza de la interrupción } & \multirow{2}{*}{ Total } \\
\cline { 2 - 4 } & Neutras & Cooperativas & Descorteses & \\
\hline \multirow{2}{*}{ Moderador hombre } & 21 & 50 & 19 & 90 \\
\cline { 2 - 4 } & $23,3 \%$ & $55,6 \%$ & $21,1 \%$ & $100,0 \%$ \\
\hline \multirow{2}{*}{ Moderador mujer } & 6 & 0 & 5 & 11 \\
\cline { 2 - 5 } & $54,5 \%$ & $0 \%$ & $45,5 \%$ & $100,0 \%$ \\
\hline \multirow{2}{*}{ Tertuliano hombre } & 0 & 8 & 190 & 198 \\
\cline { 2 - 5 } & $0 \%$ & $4,0 \%$ & $96,0 \%$ & $100,0 \%$ \\
\hline \multirow{2}{*}{ Tertuliano mujer } & 0 & 6 & 139 & 145 \\
\cline { 2 - 5 } & $0 \%$ & $4,1 \%$ & $95,9 \%$ & $100,0 \%$ \\
\hline \multirow{2}{*}{ Total } & 27 & 64 & 353 & 444 \\
\cline { 2 - 4 } & $6,1 \%$ & $14,4 \%$ & $79,5 \%$ & $100,0 \%$ \\
\hline
\end{tabular}

En ambos medios encontramos diferencias estadísticamente significativas. Tanto en televisión (96,7\% y 98,2\%), como en radio (96,0\% y 95,9\%), tertulianos y tertulianas cometen más interrupciones descorteses que los moderadores, resultado esperable dado el tipo discursivo que abordamos. En televisión, los moderadores $(54,2 \%)$ y las moderadoras $(46,2 \%)$ suelen cometer más interrupciones neutras, fruto del ejercicio de su rol en esta situación comunicativa específica. Las interrupciones cooperativas en televisión también son más frecuentes en el discurso de los moderadores, tanto hombres $(25,3 \%)$ como mujeres $(15,4 \%)$. En radio, por su parte, son los moderadores los que suelen cometer más interrupciones cooperativas (55,6\%). Las interrupciones neutras, por último, se encuentran en porcentajes más altos en el discurso de las moderadoras (54,5\%). En todos estos casos, el P valor ha sido inferior a 0,05 , en concreto, menor a 0.001 , lo que constata que nos encontramos ante diferencias altamente significativas en términos estadísticos.

Finalmente, hemos considerado oportuno observar la incidencia que las variables sexo y rol del interlocutor interrumpido tiene en el fenómeno analizado. Los datos extraídos se reflejan en la siguiente tabla:

Tabla 6. Distribución de interrupciones SEgún SU Naturaleza ATENDIENDO A LAS VARIABLES ROL Y SEXO DEL INTERLOCUTOR

\begin{tabular}{|l|l|c|c|c|c|}
\hline \multicolumn{2}{|l|}{} & Frecuencia & Porcentaje & $\begin{array}{c}\text { Porcentaje } \\
\text { válido }\end{array}$ & $\begin{array}{c}\text { Porcentaje } \\
\text { acumulado }\end{array}$ \\
\hline Válidos & Moderador hombre & 152 & 5,3 & 13,4 & 13,4 \\
\hline & Moderador mujer & 42 & 1,5 & 3,7 & 17,1 \\
\hline & Tertuliano hombre & 566 & 19,8 & 49,9 & 67,0 \\
\hline
\end{tabular}




\begin{tabular}{|l|l|c|c|c|c|}
\hline & Tertuliano mujer & 375 & 13,1 & 33,0 & 100,0 \\
\hline & Total & 1135 & 39,7 & 100,0 & \\
\hline Perdidos & Sistema & 1724 & 60,3 & & \\
\hline Total & 2859 & 100,0 & & \\
\hline
\end{tabular}

A pesar de que la inclusión de la variable rol/sexo del interlocutor interrumpido ha arrojado algunas diferencias significativas (por ejemplo, que los tertulianos son más interrumpidos por los moderadores hombres que por contertulios de ambos sexos), se trata de diferencias esperables según el tipo discursivo en el que nos encontramos. En lo relativo a las interrupciones recibidas por tertulianos o moderadores en función de su sexo, no se han registrado diferencias estadísticamente significativas.

\section{Conclusiones}

El análisis que hemos llevado a cabo nos ha permitido profundizar en el funcionamiento de la interrupción en un contexto comunicativo específico: la tertulia periodística de tema político, una interacción de naturaleza polilogal con una elevada presencia de interrupciones, hecho esperable dada la tolerancia sociocultural de los españoles a la aparición de este fenómeno (Fant, 1996).

Al realizar un análisis pragmático de la interrupción, contextualizado en el tipo discursivo de la tertulia política, se ha puesto de manifiesto la necesidad de atender a la variable rol de los hablantes implicados. La inclusión de este parámetro nos ha permitido, por una parte, constatar un mayor uso de interrupciones por parte de los tertulianos que de los moderadores y, por otra, delimitar el uso de las interrupciones de naturaleza neutra al esquema interactivo $M>T$. En lo relativo a las diferencias por medio, hemos constatado mayores índices de aparición de interrupciones totales en televisión que en radio, en el discurso de todas las instancias discursivas, a excepción de las moderadoras de radio.

Atendiendo a la naturaleza de la interrupción, se han alcanzado las siguientes conclusiones:

- En ambos medios encontramos diferencias estadísticamente significativas: tertulianos y tertulianas cometen más interrupciones descorteses que los moderadores, resultado esperable dado el tipo discursivo en el que nos encontramos.

- En televisión, los moderadores de ambos sexos producen más interrupciones neutras y cooperativas, fruto del ejercicio de su rol en esta situación comunicativa específica.

- En radio, por su parte, son los moderadores los que suelen cometer más interrupciones cooperativas. Las interrupciones neutras, por el contrario, se encuentran en porcentajes más altos en el discurso de las moderadoras. 
- No hemos constatado diferencias estadísticamente significativas atendiendo al sexo/rol del interlocutor, esto es, del hablante interrumpido.

En este sentido, ha sido más determinante la variable rol que la de sexo en los resultados extraídos, en la misma línea que las conclusiones obtenidas por Beattie $(1981)^{8}$. Este hecho constata la existencia de normas particulares en los diferentes intercambios conversacionales: en la tertulia periodística de tema político lo significativo es el rol que desempeñan los hablantes, que les permite producir o no diferentes tipos de interrupciones, mientras que el sexo no resulta un factor diferenciador. Finalmente, de este trabajo se desprende, una vez más, la necesidad de incorporar elementos extralingüísticos (rol y sexo de los hablantes implicados, tipo discursivo, intención del hablante) al análisis, lo que nos permite obtener una visión más amplia y certera de este fenómeno en cuestión.

\section{Referencias}

Ávila, A. M. (1997). El papel de las interrupciones en el desarrollo de la dinámica conversacional. En J. A. de Molina Redondo, J. de D. Luque Durán y F. Fernández García (Coords.), Estudios de lingüistica general: conferencias [y] trabajos presentados en el II Congreso Nacional de Lingüistica General, II (pp. 11-24). Granada: Método.

Bañón, A. M. (1996). La interrupción en la interacción oral entre hispanohablantes. En P. Díez de Revenga y J. M. Jiménez Cano (Eds.), Estudios de sociolingüistica. Sincronía $y$ diacronía (pp. 130-154). Murcia: DM.

Bañón, A. M. (1997). La interrupción conversacional. Propuesta para su análisis pragmalingüistico. Málaga: Analecta Malacitana.

Beattie, G. (1981). Interruption in conversational interaction and its relation to the sex and status of the interactans. Linguistics, 19(1-2), 15-35.

Bengoechea, M. (1993). La interrupción entre jefas y subordinadas/os. En J. FernándezBarrientos Martín (Ed.), Jornadas Internacionales de Lingüistica Aplicada, II (pp. 1520). Granada: Universidad de Granada.

Bolívar, A. (2010). ¿Por qué no te callas? La función de las interrupciones en el diálogo político. En I. Fonte Zarabozo y L. Rodríguez Alfano (Comps.), Perspectivas dialógicas en estudios del lenguaje (pp. 299-336). México: Universidad de Nuevo León.

Bravo, D., y Briz, A. (Eds.) (2004). Pragmática sociocultural: Estudios sobre el discurso de cortesía en español. Barcelona: Ariel.

Bresnahan, M., y Cai, D. (1996). Gender and agression in the recognition of interruption. Discourse Processes, 21, 171-189.

8. Según el cual, a partir del análisis de diez intercambios en tutorías universitarias, se constata que, mientras que el sexo no parece tener influencia en la aparición de procesos interruptores, el estatus afecta inversamente: a más estatus del hablante menos interrupciones (Beattie, 1981). 
Briz, A. (1998). El español coloquial en la conversación. Esbozo de pragmagramática. Barcelona: Ariel.

Carbó, T. (1992). Towards an interpretation of interruptions in Mexican parliamentary discourse (1920-1960). Discourse \& Society, 3(1), 25-45.

Cestero, A. M. (1994a). Intercambios de turnos de habla en la conversación en Lengua Española. Revista de la Sociedad Española de Lingüistica, 24, 77-99.

Cestero, A. M. (1994b). Alternancia de turnos de habla en lengua española: la influencia del sexo y la edad de los interlocutores. Pragmalingüistica, 2, 123-150.

Cestero, A. M. (2007). Cooperación en la conversación: Estrategias estructurales características de las mujeres. LinRed. Lingüistica en la red, V, 1-17. Recuperado de http://www.linred.es/articulos_pdf/LR_articulo_24042007.pdf.

Cordisco, A. (2003). Afiliación y desafiliación: Contexto sociocultural en el análisis de la interrupción y de sus consecuencias sociales en la interacción. En D. Bravo (Ed.), Actas del Primer Coloquio del Programa EDICE "La perspectiva no etnocentrista de la cortesía: identidad sociocultural de las comunidades hispanohablantes" (pp. 149163). Estocolmo: Universidad de Estocolmo. Recuperado de http://www.edice.org/ descargas/1 coloquioEDICE.

Duncan, S. Jr. (1972). Some signals and rules for taking speaking turns in conversations. Journal of Personality and Social Psychology, 23(2), 283-293.

DXSP-OPS = Dirección Xeral de Saúde Pública (Xunta de Galicia) - Organización Panamericana de la Salud.

DXSP-OPS (2006). Epidat 3.1 para WIndows. La Coruña: Xunta de Galicia.

Fant, L. (1989). Cultural mismatch in conversation: Spanish and Scandinavian communicative behavior in negotiation settings. Hérmes, 2, 247-265.

Fant, L. (1996). Regulación conversacional en la negociación: Una comparación entre pautas mexicanas y peninsulares. En T. Kotschi, W. Oesterreicher y K. Zimmermann (Eds.), El español hablado y la cultura oral en España e Hispanoamérica (pp. 147-185). Frankfurt/ Madrid: Vervuert/Iberoamericana.

Fuentes, C. (2000). Lingüistica pragmática y análisis del discurso. Madrid: Arco/Libros.

Fraser, B., y Nolan, W. (1981). The association of deference with linguistic form. International Journal of the Sociology of Language, 27, 93-111.

Gallardo, B. (1993). La transición entre turnos conversacionales: Silencios solapamientos e interrupciones. Contextos, XI(21-22), 189-220.

García Mouton, P. (1999). Cómo hablan las mujeres. Madrid: Arco/Libros.

Goldberg, J. (1990). Interrupting the discourse on interruptions. An analysis in terms of relationally neutral power-and rapport- oriented acts. Journal of Pragmatics, 14(6), 883-903.

González-Sanz, M. (2014). Tertulia política y descortesía verbal. Tesis Doctoral. Universidad de Sevilla. doi:10.13140/RG.2.1.1605.2724

González-Sanz, M. (2017). Tertulia e ideología. La mediatización del pensamiento politico. Berna: Peter Lang. 
Hansen, S. B. (1997). Talking about politics: Gender and contextual effects on political proselytizing. Journal of Politics, 59, 73-103.

Haverkate, H. (1994). La cortesía verbal. Estudio pragmalingüistico. Madrid: Gredos.

Hidalgo, A. (1998). Alternancia de turnos y conversación. Sobre el papel regulador de los suprasegmentos en el habla simultánea. Lingüistica Española Actual, 20(2), 218238.

Holmes, J. (1995). Women, men and politeness. Londres: Longman.

Hutchby, I. (1992). Confrontation talk: Aspects of 'interruption' in argument sequences on talk radio. Text, 12, 343-371.

James, D., y Clarke, S. (1993). Women, men and interruptions. En D. Tannen (Ed.), Gender and conversational interaction (pp. 231-280). Nueva York/Oxford: Oxford University Press.

Johnson, F. (2006). Agreement and disagreement: A cross-cultural comparison. BISAL, 1, 41-67.

López Serena, A., y Méndez, E. (2009). La interrupción como mecanismo regulativo de las interacciones verbales. Los debates electorales Zapatero-Rajoy 2008. Español Actual, 92, 159-220.

Lozano, I. (1995). Lenguaje femenino, lenguaje masculino. ¿Condiciona nuestro sexo la forma de hablar? Madrid: Minerva Ediciones.

Murata, K. (1994). Intrusive or cooperative? A cross-cultural study of interruption. Journal of Pragmatics, 21, 385-400.

Poyatos, F. (1980). Interactive functions and limitations of verbal and non verbal behavior in natural conversations. Semiótica, 30(3/4), 211-244.

Ridao Rodrigo, S. (2009). Estrategias de (des)cortesía en las mediaciones laborales. Murcia: Editum, Ediciones de la Universidad de Murcia.

Roger, D., Bull, P., y Smith, S. (1988). The development of a comprehensive system for classifying interruptions. Journal of Language and Social Psychology, 7(1), 27-34.

Sacks, H., Schegloff, E. A., y Jefferson, G. (1974). A simplest systematics for the organization of turn-talking for conversation. Language, 50(4), 696-735.

Tannen, D. (1994). Interpreting interruption in conversation. En D. Tannen (Ed.), Gender and discourse (pp. 63-90). Oxford: Oxford University Press.

Watts, R. J. (1991). Power in family discourse, Berlin: De Gruyter.

West, C., y Zimmerman, D. H. (1987). Small insults: A study of interruptions in cross-sex conversation between unacquainted persons. En B. Thorne, C. Kramarae y N. Henley (Eds.), Language gender and society (pp. 103-117). Cambridge: MA, Newbury House.

Zimmerman, D. H., y West, C. (1975). Sex roles, interruptions and silences in conversation. En B. Thorne y N. Henley (Eds.), Language and sex: Deference and dominance (pp. 105-129). Rowley, Mass: Newbury House 


\section{Apéndice: Convenciones de transcripción}

Nombre Apellido:

$\$$

$=$

[

$-$

I

$/ /$

/II

$\uparrow$

$\downarrow$

$\rightarrow$

Partido Popular

PESADO

pe sa do

pa'l

${ }^{\circ}()^{\circ}$

S

aa

nn

¿i!?

i?

¿eh?,

i!
Intervención de un interlocutor identificado en la interacción. Sucesión inmediata, sin pausa apreciable, entre dos emisiones de distintos interlocutores.

Mantenimiento del turno de un participante en un solapamiento.

Lugar donde se inicia un solapamiento o superposición.

Final del habla simultánea.

Reinicios y autointerrupciones sin pausa.

Pausa corta, inferior al medio segundo.

Pausa entre medio segundo y un segundo.

Pausa de un segundo o más.

Entonación ascendente.

Entonación descendente.

Entonación mantenida o suspendida.

Los nombres propios, apodos, siglas y marcas, excepto las convertidas en "palabras-marca» de uso general, aparecen con la letra inicial en mayúscula.

Pronunciación marcada o enfática (dos o más letras mayúsculas).

Pronunciación silabeada.

Fenómenos de fonética sintáctica entre palabras, especialmente marcados.

Fragmento pronunciado con una intensidad baja o próxima al usurro.

Alargamientos vocálicos.

Alargamientos consonánticos.

Interrogaciones exclamativas.

Interrogaciones. También para los apéndices del tipo ¿no?, ¿sabes?

Exclamaciones. 